\title{
Electronic Detection of Oxygen Adsorption and Size-Specific Doping of Few-Atom Gold Clusters on Graphene
}

\author{
Jeroen E. Scheerder, ${ }^{1}$ Shuanglong Liu,${ }^{2}$ Vyacheslav S. Zharinov, ${ }^{1}$ Nicolas Reckinger, ${ }^{3}$ \\ Jean-François Colomer, ${ }^{3}$ Hai-Ping Cheng, ${ }^{2}$ Joris Van de Vondel,,${ }^{1, *}$ and Ewald Janssens ${ }^{1, \dagger}$ \\ ${ }^{1}$ Laboratory of Solid-State Physics and Magnetism, \\ KU Leuven, Celestijnenlaan 200D, 3001 Leuven, Belgium \\ ${ }^{2}$ Department of Physics and the Quantum Theory Project, \\ University of Florida, Gainesville, FL 32611 \\ ${ }^{3}$ Research Group on Carbon Nanostructures (CARBONNAGe), \\ University of Namur, Rue de Bruxelles 61, 5000 Namur, Belgium.
}

\begin{abstract}
Graphene's sensitivity to adsorbed particles has attracted widespread attention because of its potential sensor applications. Size-selected few-atom clusters are promising candidates as adparticles to graphene. Due to their small size, physico-chemical properties are dominated by quantum size effects. In particular, few-atom gold clusters demonstrate a significant catalytic activity in various oxidation reactions. In this joint experimental and computational work, we investigate size-selected gold clusters with 3 and 6 atoms adsorbed on graphene field-effect transistors and their interaction with molecular oxygen. While oxygen adsorbs at both cluster sizes, there is a pronounced cluster size-dependence in the corresponding doping, as demonstrated via first-principles calculations and electronic transport measurements. Furthermore, the doping of gold cluster decorated graphene changes sign from $n$ - to $p$-doping upon oxygen adsorption, directly evidencing electron transfer to the oxygen molecules and hence their activation. From these observations, graphene promises to be a valuable platform to investigate and exploit size-dependent cluster properties.
\end{abstract}

Keywords: Graphene; field-effect transistor; few-atom gold cluster; oxygen adsorption and activation; DFT 
More than 10 years ago, the discovery of graphene's properties ignited research on twodimensional (2D) materials, enabling new and exciting opportunities in both fundamental and applied sciences. The ultimate confinement in one dimension enables gate control of graphene's transport properties. ${ }^{[1]}$ Moreover, graphene is very sensitive to materials in its vicinity, which can either be the supporting substrate, ${ }^{[2]}$ adsorbed particles (adparticles), ${ }^{[3,4]}$ or stacks of other (2D) materials. ${ }^{[5,6]}$ It is this susceptibility to its direct environment which allowed to explore graphene-based devices as a particle sensor, relying on either its mechanical ${ }^{[7-9]}$ or electronic properties ${ }^{[10,11]}$ and to foster desired (electronic) properties in graphene, such as (tunable) bandgaps ${ }^{[12]}$ or $p-n$ junctions. $^{[13]}$

Metal atoms and nanoparticles are interesting candidates to tailor graphene. ${ }^{[14]}$ The charge transfer between adparticles and graphene results in tunable (surface) electronic states, which can act as active sites for heterogeneous catalysis, ${ }^{[4,15-17]}$ or enhance the sensitivity and selectivity of graphene gas sensors (see Ref. 18 and references therein). Furthermore, metal adparticles are prime candidates to induce a (tunable) spin-orbit coupling in graphene, enhancing for instance the spin Hall effect ${ }^{[19]}$ which further augments graphene's spintronic potential ${ }^{[20]}$.

Due to the extreme sensitivity of graphene devices, one desires a high level of control in adsorbing metal adparticles. Such control is offered by state-of-the-art cluster fabrication and deposition techniques, which allows to select the size and composition of clusters with atomic resolution, and tune the deposition energy and adparticle density. ${ }^{[21]}$ Using these techniques, ultra-small few-atom clusters in gas-phase showcased a distinct atom-by-atom size-dependence in the electronic and structural properties, leading to different and unique physico-chemical properties. ${ }^{[22]}$.

The size-dependent characteristics can be preserved in the interaction of a cluster with a support. For specific gold, cobalt and germanium clusters, dedicated atomic resolution surface probe studies, using scanning tunnelling microscopy ${ }^{[23,24]}$ and scanning transmission electron microscopy ${ }^{[25-28]}$, have, in combination with Density Functional Theory (DFT) simulations, allowed for a detailed morphological characterization of clusters on supports. The overall properties of a cluster-support system retain a dependence on the exact cluster size. ${ }^{[15]}$ As such, cluster-support systems, engineered with atomic precision, are, among others, of interest as catalysts ${ }^{[29-32]}$ and low-reactive building blocks for nanosystems. ${ }^{[33]}$ In the size-regime in between single atoms and larger nanometre-sized particles, clusters offer 
diverse possibilities in functionalizing graphene.

To the best of our knowledge, there has been no realisation yet of an electronic device, in which the rich size-dependence of few-atom metal clusters is transpired in the properties of the device, although this has been proposed in several computational studies for few-atom metal clusters on graphene. ${ }^{[34-36]}$ To that avail, we combine in this work single layer graphene $(\mathrm{G})$ with few-atom gold clusters. In particular, these clusters show a high and size-dependent reactivity, which stands in stark contrast with the inertness of bulk gold. Haruta et al. ${ }^{[37]}$ first observed the remarkable catalytic activity of gold nanoparticles, notably towards oxidation reactions involving molecular oxygen. This has been extended to few-atom $\mathrm{Au}_{m}(m \lesssim 20)$ clusters in gas-phase studies, illuminating their atom-to-atom size-dependent affinity for oxygen adsorption and dissociation, relevant reaction pathways, and the dependence of the reactivity on the initial charge state of the cluster. ${ }^{[38-42]}$

Here we investigate the adsorption of $\mathrm{Au}_{m}(m=3,6)$ on graphene and the interaction of the resulting $\mathrm{Au}_{m} / \mathrm{G}$ hybrid system with molecular oxygen $\left(\mathrm{O}_{2}\right)$. The latter is inspired by studies on few-atom gold clusters, both in gas-phase and on supports, which have identified the binding of molecular oxygen to gold clusters ${ }^{[38-40]}$ as a model system for in oxidation reactions. Stable adsorption of $\mathrm{O}_{2}$ is found for both cluster sizes, but the characteristics of the resulting $\mathrm{O}_{2} / \mathrm{Au}_{m} / \mathrm{G}$ systems are dictated by the cluster's size and are detectable in electronic transport measurements. The experimental findings are in agreement with complementary DFT simulations.

The experimental methodology relies on a novel combination of size-selected cluster deposition and in situ field-effect measurements in graphene (i.e. inside the ultra high vacuum (UHV) cluster deposition chamber), in which there is size selection with atomic resolution and a high level of control over deposition energy and adparticle density. ${ }^{[21]}$ To study the interaction with $\mathrm{O}_{2}$, the experimental setup is further augmented with controlled in situ gas insertion. The $\mathrm{Au}_{m}$ cluster ions are produced in a home-built magnetron sputtering setup and size selected by a quadrupole mass-filter (see Experimental Section). In Fig. 1a is shown $\mathrm{a} \mathrm{Au}_{m}(m=1-7)$ mass spectrum, demonstrating the achieved atomic resolution. The field-effect characteristic of a representable virgin (i.e. as-processed) graphene fieldeffect transistor (GFET) is shown in Fig. 1b. The inset presents a false coloured scanning electron micrograph of a representable GFET device. The graphene transport channel, fa- 

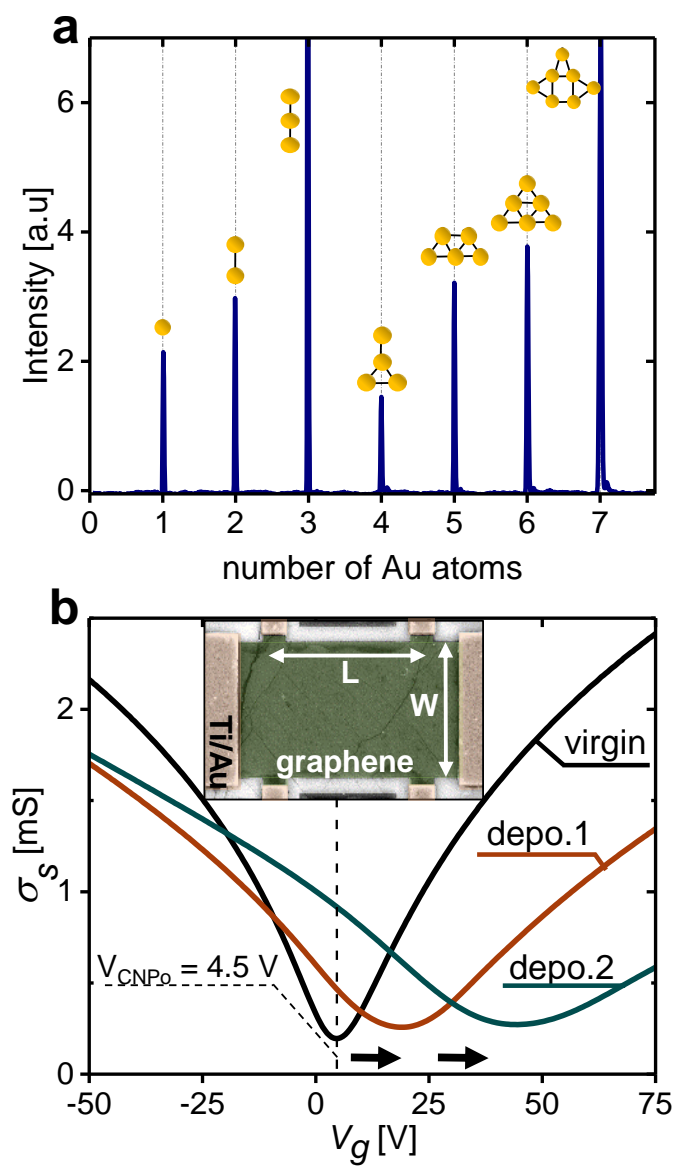

FIG. 1: Overview of the experimental approach. a) High resolution mass spectrum of $\mathrm{Au}_{n}$ anions $(n=1-7)$, with the insets showing their structure as determined in Ref. 43. b) Field-effect characteristic $\sigma_{s}\left(V_{g}\right)$ for a virgin device and after two consecutive depositions (depo.1 and depo.2,) in which $\mathrm{Au}_{3}$ /oxygen complexes are introduced. The charge neutrality point of the virgin device, $V_{C N P o}$, is indicated. In the inset is shown a false coloured scanning electron microscopy image of a representable GFET device with width $W=10 \mu \mathrm{m}$ and length $L=12 \mu \mathrm{m}$.

bricated from single-layer chemical vapour deposition (CVD) grown graphene, ${ }^{[44]}$ has length $L=12 \mu \mathrm{m}$ and width $W=10 \mu \mathrm{m}$ and is contacted by $30 \mathrm{~nm}$ Au contacts with a Ti adhesion layer and it is supported on a $p^{++} \mathrm{Si} / \mathrm{SiO}_{2}(300 \mathrm{~nm})$ substrate. The sheet conductance $\sigma_{s}$ $[\mathrm{mS}]$ is shown as a function of the gate voltage $V_{g}[\mathrm{~V}]$ applied between the graphene channel and the backgate $p^{++}$Si electrode. By sweeping the gate voltage, charge carriers are induced and as such, graphene's Fermi level can be altered, resulting in the well-known ambipolar field-effect behaviour. ${ }^{[45]}$ The minimum in conductivity occurs at the charge neutrality point 
(CNP), or $V_{g}=V_{C N P}$, and the electronic transport is governed by electrons (holes) for $V_{g}>V_{C N P}\left(V_{g}<V_{C N P}\right)$ since the Fermi level is then located in the conduction (valence) band. The charge neutrality point of the virgin device, $V_{C N P o}$, equals $4.5 \mathrm{~V}$. This indicates an unintentional mild $p$-doping resulting from the device fabrication. The detection of adsorbed particles is illustrated in Fig. 1b by two field-effect characteristics of graphene decorated with $\mathrm{Au}_{3}$-oxygen complexes (with the coverage of depo.2 being higher than depo.1). Two clear effects arise: i) the CNP shifts to higher values of $V_{g}$, indicating $p$-doping, and ii) the slope of the $\sigma_{S}\left(V_{g}\right)$ characteristic becomes smaller, indicating a decrease of the charge carrier mobility (i.e increased scattering). Both effects can be linked to the charge transfer between the adsorbed $\mathrm{Au}_{3}$-oxygen complexes and graphene, as will be discussed more in detail below.

When depositing size-selected charged clusters on the graphene device, the net charge of the cluster will flow away through the device, since it is grounded during deposition. The resulting current is used to determine with appreciable accuracy the density of deposited clusters $n_{c}\left[\mathrm{~cm}^{-2}\right] .{ }^{[21]}$ The (neutralised) adsorbed cluster interacts with the graphene, resulting in partial charge transfer, as shown schematically in Fig. 2a. The irradiation of the device with a charged particle beam induces additional effects which are not related to the cluster-graphene interaction. ${ }^{[21]}$ As demonstrated in Supporting Note 1 those unwanted effects neutralise on a time scale of several days in the UHV environment. Lowering the impact energy of the deposited clusters significantly reduces those effects, at the drawback of a lower cluster flux.

Fig. $2 \mathrm{~b}$ shows the shift in charge neutrality point with respect to the virgin sample $\Delta V_{C N P}=V_{C N P}-V_{C N P o}$ for a device decorated with $\mathrm{Au}_{3}^{-}$clusters with a minimal deposition energy of $\sim 3 \mathrm{eV}$ by decelerating them with a repelling voltage on the device. $\Delta V_{C N P}$ was measured in function of time in the UHV deposition chamber for a period of roughly 3 days. $t=0 \mathrm{~min}$ corresponds to the end of the deposition, and $n$-doping (i.e. $\Delta V_{C N P}<0 \mathrm{~V}$ ) is retrieved. When monitoring the charge neutrality point in function of time, we see an insignificant change in time and attribute the observed shift $\left(\Delta V_{C N P, a v}=(-11.4 V \pm 0.8)\right.$ V) to doping of graphene by the deposited $\mathrm{Au}_{3}$ clusters.

In order to quantify the amount of doping from $\Delta V_{C N P}$, one can employ the doping efficiency $\varepsilon,{ }^{[46]}$ i.e. the amount of induced charge (either electrons $e$ or holes $h$ for $n$ - or p-doping, respectively) in graphene per deposited cluster (see Experimental Section). In 

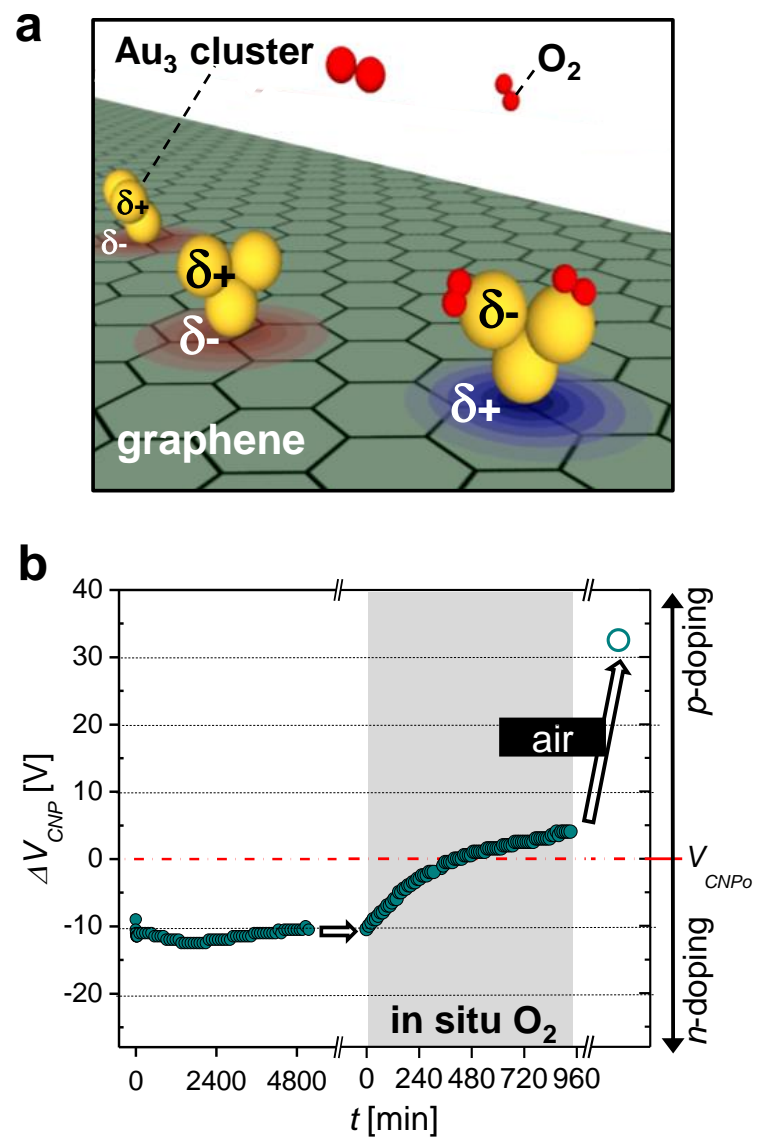

FIG. 2: a) Schematic representation of $\mathrm{Au}_{3}$ on graphene. The partial charge transfer between the clusters and graphene is indicated. Molecular oxygen can adsorb on the $\mathrm{Au}_{3}$ cluster sites, resulting in a sign change of the charge transfer. b) Time evolution of $\Delta V_{C N P}$ after deposition of $\mathrm{Au}_{3}^{-}$with a density $n_{c}=(17 \pm 1) \cdot 10^{13} \mathrm{~cm}^{-2}$. The device is then in situ exposed to $\mathrm{O}_{2}$ at a pressure of $10^{-4}$ mbar (grey shaded area). After in situ exposure to $\mathrm{O}_{2}$, the sample was exposed to air. After re-installation in the UHV chamber, $\Delta V_{C N P}=32.5 \mathrm{~V}$ (open symbol).

Ref. 21, it was found that the majority of the deposited clusters remain on graphene as either single clusters, while the minority coalesces into bigger entities of a few nanometre ${ }^{[47]}$, that do not contribute significantly to the doping. Hence, for estimation of $\varepsilon$ we employ the best estimate for the non-coalesced cluster density $n_{C}^{\dagger}$. For the depositions in this paper, $n_{C}^{\dagger} / n_{C}=(68 \pm 12) \%$ and $n_{C}^{\dagger} / n_{C}=(61 \pm 11) \%$ for $\mathrm{Au}_{3}$ and $\mathrm{Au}_{6}$ decorated samples, respectively. Using $\Delta V_{C N P, a v}$ we can estimate the experimental doping efficiency of $\mathrm{Au}_{3}$ on graphene: $\varepsilon_{\mathrm{Au}_{3}}^{\mathrm{exp}}=0.007 \pm 0.002 \mathrm{e} /$ cluster. This estimate is in good agreement with DFT 
calculations, both in sign and amplitude, as is shown below.

Subsequently, the $\mathrm{Au}_{3} / \mathrm{G}$ system is exposed to oxygen. Figure 2b (grey shaded area) depicts the evolution of the CNP (and hence doping) as a function of time when the $\mathrm{Au}_{3} / \mathrm{G}$ device is in situ exposed to $\mathrm{O}_{2}$ gas at room temperature and pressure $\mathrm{P} \sim 10^{-4}$ mbar. The CNP increases, eventually to a net $p$-doped state (i.e. with respect to the virgin sample $\Delta V_{C N P}>0 \mathrm{~V}$ ). The device is brought into ambient conditions (air exposure at 1 bar) after $935 \mathrm{~min}$ of exposure to oxygen (a dosage of $\sim 4.2$ Torr $\cdot \mathrm{s}=4.2 \cdot 10^{6} \mathrm{~L}$ ). After inserting the sample back in the UHV chamber, we retrieve a $p$-doped state with $\Delta V_{C N P}=32.5 \mathrm{~V}$ $\left(\varepsilon_{\mathrm{O}_{2} / \mathrm{Au}_{3}}^{\exp }=(0.020 \pm 0.004) h /\right.$ cluster $)$. The shift of $V_{C N P}$ can be attributed to the binding of molecular oxygen to the $\mathrm{Au}_{3} / \mathrm{G}$ system, as sketched in Fig. 2a, which is surprisingly stable at room temperature as demonstrated by the DFT calculations below. It is the adsorption of oxygen on the $\mathrm{Au}_{3}$ cluster sites that causes the observed p-doping. Note that oxygen adsorption on virgin graphene devices results in doping with opposite sign (see Supporting Note 2). This evidences that the Au clusters drastically influence the oxygen adsorption. Reference in situ gas exposure measurements using inert Ar gas gave the expected null result.

The findings shown in Fig. 2 are in agreement with our first-principles calculations of $\left(\mathrm{O}_{2} /\right) \mathrm{Au}_{m} / \mathrm{G}$ systems. In Fig. 3a and $\mathrm{b}$ are shown the computationally lowest energy configurations for both $\mathrm{Au}_{3}$ and $\mathrm{Au}_{6}$ clusters on a graphene unit cell of $14.77 \AA \times 14.77 \AA$ (see Experimental Section). The respective cluster binding energies are $-1.23 \mathrm{eV}$ and -1.25 $\mathrm{eV}$, which indicates that both species have a large adsorption energy to graphene.

For free (neutral) $\mathrm{Au}_{3}$ clusters, one has observed two isomers which are close in energy (energy difference on the order of $20 \mathrm{meV}$ ). ${ }^{[41,48]}$ One isomer has a triangular geometry, while the other isomer portrays a more linear shape. Upon adsorption to graphene, we find that a structure very similar to the triangular free cluster isomer is energetically preferred, with only a small change in the Au-Au bond lengths $(\lesssim 0.05 \AA)$ and angles $\left(\lesssim 2^{\circ}\right)$ (see Supporting Note 3.5), whereas a more linear $\mathrm{Au}_{3} / \mathrm{G}$ geometry is calculated to be $0.37 \mathrm{eV}$ higher in energy (see Supporting Note 3.4). The triangular $\mathrm{Au}_{3}$ adsorbs vertically on a graphene bridge site, where the anchor gold atom has two nearest-neighbour carbon atoms with $\mathrm{Au}-\mathrm{C}$ distances of $2.31 \AA$.

The adsorbed $\mathrm{Au}_{6}$ has a very similar planar structure as its neutral free cluster counterpart ${ }^{[41,48]}$, with a minor change in bond lengths $(\lesssim 0.01 \AA)$ and bond angles $\left(\lesssim 0.05^{\circ}\right)$ 
(see Supporting Note 3.5). $\mathrm{Au}_{6}$ adsorbs parallel to the graphene plane at an average distance of $3.29 \AA$ (Fig. 3b). Other configurations have been tested for both $\mathrm{Au}_{3,6} / \mathrm{G}$ and are shown in Supporting Note 3.4. In particular for $\mathrm{Au}_{6}$, the difference in adsorption energy of these configurations is lower than the thermal energy $k_{B} T$ at room temperature. Based on this, we expect the coexistence of multiple adsorption configurations on graphene and possible thermally driven dynamics of the system. For $\mathrm{Au}_{3} / \mathrm{G}$, the second-lowest-energy configuration lies higher in energy by $50 \mathrm{meV}$, so a small thermal population might be possible.

Figures $3 \mathrm{c}$ and $3 \mathrm{~d}$ depict the geometry of the lowest-energy $\mathrm{O}_{2} / \mathrm{Au}_{m} / \mathrm{G}$ systems $(m=$ $3,6)$. Note that four oxygen molecules (three oxygen molecules) adsorbed on the $\mathrm{Au}_{3}\left(\mathrm{Au}_{6}\right)$ cluster are the maximum amounts that are thermally stable. However, the conclusions of this work do not alter for a different number of oxygen molecules per cluster (see Supporting Note 3.2). The orientation of the metal clusters is similar as in Figures 3a en 3b. However, the anchor atom in $\mathrm{Au}_{3}$ shifts laterally to be atop a $\mathrm{C}$ atom, forming a single $\mathrm{Au}-\mathrm{C}$ bond with length $2.28 \AA$. The average distance from the gold atoms of $\mathrm{Au}_{6}$ to the graphene plane is $3.31 \AA$, slightly larger than for the $\mathrm{Au}_{6} / \mathrm{G}$ system. Upon oxygen adsorption, the $\mathrm{Au}-\mathrm{Au}$ bonds undergo small changes in length $(\lesssim 0.07 \AA)$ and bond angles $\left(\lesssim 2^{\circ}\right)$ for both $\mathrm{Au}_{3} / \mathrm{G}$ and $\mathrm{Au}_{6} / \mathrm{G}$ (see for details Supporting Note 3.5).

Other oxygen adsorption sites have been considered by investigating multiple configurations of $\mathrm{O}_{2} / \mathrm{Au}_{m} / \mathrm{G}(m=3,6)$ with a single oxygen molecule. The results are presented in Supporting Note 3.6. For $\mathrm{Au}_{6}$, the $\mathrm{O}_{2}$ molecules prefer to adsorb at a vertex site, since the relative binding energy of a different type of site is $\geq 0.45 \mathrm{eV}$. This configuration is comparable to oxygen adsorbed on isolated (neutral) $\mathrm{Au}_{6}$ clusters. ${ }^{[41,42]}$ For an isolated (neutral) $\mathrm{Au}_{3}$ cluster, it has been observed that the $\mathrm{O}_{2}$ molecule adsorbs at a bridge position, forming two $\mathrm{Au}-\mathrm{O}$ bonds, ${ }^{[41,42]}$ which differs from the most stable $\mathrm{O}_{2} / \mathrm{Au}_{3} / \mathrm{G}$ configuration. We find that the lowest-energy configuration has the $\mathrm{O}_{2}$ molecule attached to a vertex site with a single Au-O bond, while the configuration with a bridging $\mathrm{O}_{2}$ molecule is $0.5 \mathrm{eV}$ less stable (see Supporting Note 3.6).

The O-O bond length varies with the adsorption site. As labelled in Fig. 3c and 3d, the $\mathrm{O}_{2}$ bond lengths are $l_{1}=l_{3}=1.24 \AA, l_{2}=1.29 \AA, l_{4}=1.28 \AA$ and $l_{5}=l_{6}=l_{7}=1.27 \AA$, which means all oxygen bonds, except for $l_{1}$ and $l_{3}$, are significantly elongated relative to the calculated bond length for free molecular oxygen of $1.23 \AA$. This elongation indicates activation of the adsorbed oxygen molecules. The average adsorption energy of the oxygen 

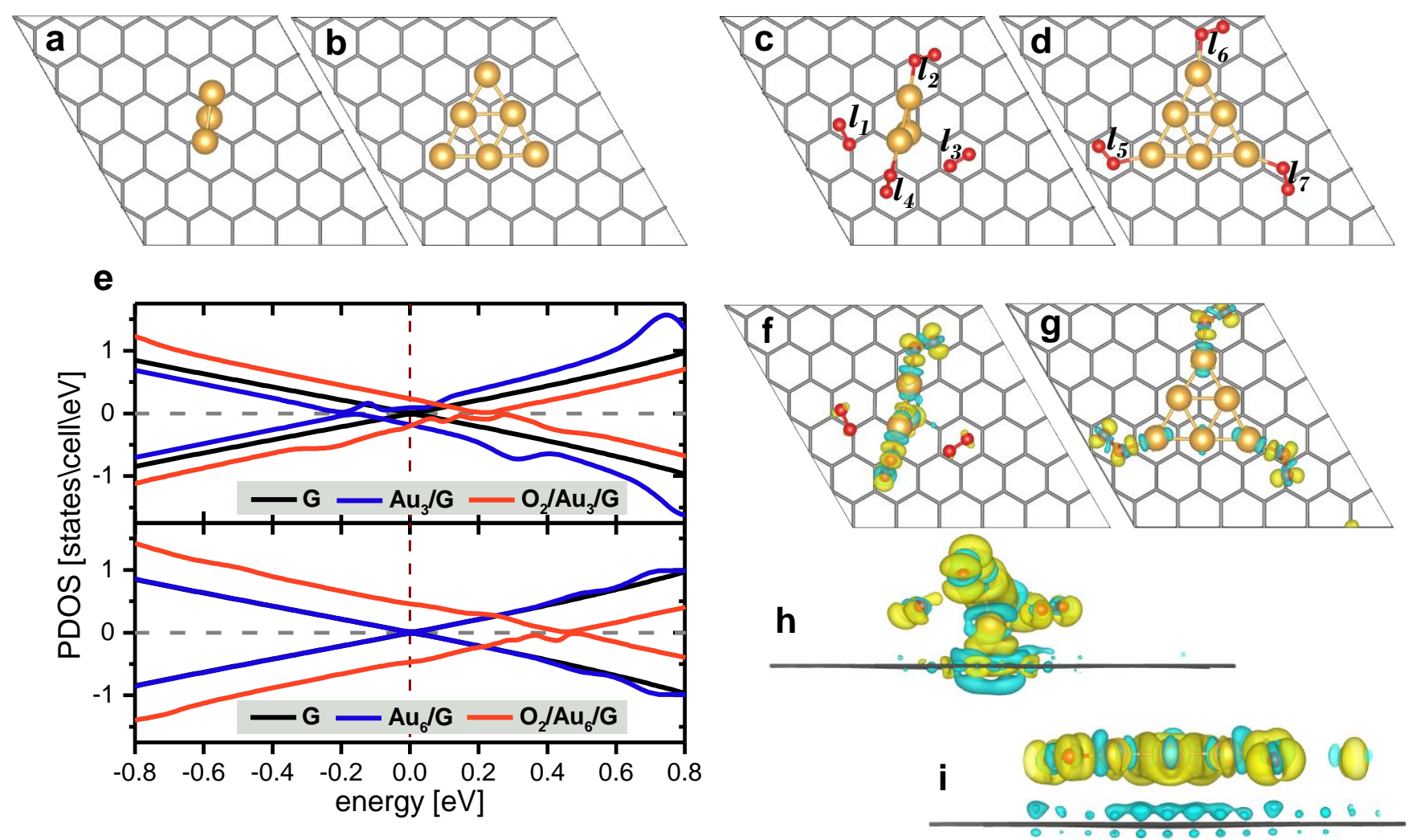

FIG. 3: DFT computations of $\mathrm{Au}_{3,6}$ on graphene, both with and without the adsorption of molecular oxygen. In panels $\mathbf{a}$ and $\mathbf{b}$ are shown the relaxed atomic configurations of $\mathrm{Au}_{3}$ and $\mathrm{Au}_{6}$ on graphene, respectively. Panels $\mathbf{c}$ and $\mathbf{d}$ depict the relaxed configurations of $\mathrm{Au}_{3}$ and $\mathrm{Au}_{6}$ on graphene with respectively 4 and 3 adsorbed $\mathrm{O}_{2}$ molecules. In e the PDOS on the $p_{z}$ orbitals of pristine graphene is compared to that of the system with (oxidized) $\mathrm{Au}_{3}$ clusters (top) and (oxidized) $\mathrm{Au}_{6}$ clusters (bottom) on graphene. Positive (negative) values of the PDOS denote spin up (down) electrons. The shift of the Dirac point with respect to pristine graphene demonstrates a significant $n$-doping for $\mathrm{Au}_{3} / \mathrm{G}$, and $p$-doping for both $\mathrm{O}_{2} / \mathrm{Au}_{3,6} / \mathrm{G}$. For $\mathrm{Au}_{6} / \mathrm{G}$, the computated doping is close to zero. f,g Charge density difference for the $\mathrm{O}_{2} / \mathrm{Au}_{3,6} / \mathrm{G}$ systems, defined as $\delta \rho=\rho_{\mathrm{O}_{2} / \mathrm{Au}_{3} / \mathrm{G}}-\rho_{\mathrm{O}_{2}}-\rho_{\mathrm{Au}_{3}}-\rho_{\mathrm{G}}$ with isovalue $3 \cdot 10^{-3} \mathrm{Bohr}^{-3}$. Yellow (cyan) colour denotes a positive (negative) $\delta \rho$, indicating a gain (loss) of electrons. Panels $\mathbf{h}$ and $\mathbf{i}$ depict the side view of panel $\mathbf{f}$ and $\mathbf{g}$ respectively with isovalue $6 \cdot 10^{-4} \mathrm{Bohr}^{-3}$. The smaller isovalue in the latter case is employed to visualize the charge transfer near the graphene plane (full black line).

molecules are $-0.55 \mathrm{eV}$ for $\mathrm{Au}_{3}$ and $-0.49 \mathrm{eV}$ for $\mathrm{Au}_{6}$, indicating stable adsorption. These values are much larger than the adsorption energy of an isolated oxygen molecule on pristine 
graphene, which is $-0.22 \mathrm{eV}$. Therefore, both $\mathrm{Au}_{3}$ and $\mathrm{Au}_{6}$ clusters assist in the adsorption of oxygen at room temperature.

If a single $\mathrm{O}_{2}$ molecule is adsorbed, the binding energies for $\mathrm{O}_{2} / \mathrm{Au}_{3} / \mathrm{G}$ and $\mathrm{O}_{2} / \mathrm{Au}_{6} / \mathrm{G}$ are $-1.12 \mathrm{eV}$ and $-0.55 \mathrm{eV}$, respectively. The adsorption energy of $\mathrm{O}_{2}$ to (neutral) $\mathrm{Au}_{3}$, as reported in Ref.'s 38 and 42, is slightly lower than $-0.6 \mathrm{eV}$ and the adsorption energy to $\mathrm{Au}_{6}$ is slightly lower than $-0.2 \mathrm{eV}$. The system portrays adsorption enhancement due to interactions among the subsystems (i.e. the interaction is not local). Similar enhanced adsorption was previously observed in $\mathrm{O}_{2} / \mathrm{N}_{2} / \mathrm{Ag}_{m}$ cluster complexes ${ }^{[49]}$. For the first time, this enhancement is observed on a graphene surface.

We furthermore investigated the possible spin transition involved in the adsorption of a single $\mathrm{O}_{2}$ molecule. For free neutral clusters, as described in detail in Ref. 42, the interaction of a triplet $\mathrm{O}_{2}$ molecule with a doublet $\mathrm{Au}_{3}$ or a singlet $\mathrm{Au}_{6}$ leads to doublet $\mathrm{O}_{2} / \mathrm{Au}_{3}$ or triplet $\mathrm{O}_{2} / \mathrm{Au}_{6}$, respectively. Hence, the spin transition is allowed for $\mathrm{Au}_{3}$ and there is no spin transition for $\mathrm{Au}_{6}$. For the final states $\mathrm{O}_{2} / \mathrm{Au}_{3} / \mathrm{G}$ and $\mathrm{O}_{2} / \mathrm{Au}_{6} / \mathrm{G}$, we calculated spin magnetic moments of $1.00 \mu_{B}$ and $1.77 \mu_{B}$, respectively, located on the adsorbed $\mathrm{O}_{2}$ molecule, and the calculations reveal comparable reaction pathways to the free cluster cases. As such, for both sizes, the process seems spin allowed and no significant barriers are involved. Earlier work on gas phase clusters ${ }^{[42]}$ also demonstrated that after $\mathrm{O}_{2}$ adsorption, large barriers (more than $2 \mathrm{eV}$ ) are involved in dissociation of the oxygen molecule, so this process was not considered in the present study.

Figure 3e presents the calculated projected density of states (PDOS) on the atomic $p_{z}$ orbital of carbon atoms, which are the orbitals carrying electrical current, for $\left(\mathrm{O}_{2}\right) / \mathrm{Au}_{3,6} / \mathrm{G}$ and pristine graphene. One can recognize the Dirac point as the point where the PDOS vanishes. For pristine graphene, the Dirac point $E_{D}$ coincides with the Fermi level $E_{F}$, where the latter has been set to $0 \mathrm{eV}$ on the energy scale. Upon doping with $\mathrm{Au}_{3}$ clusters, the Dirac point shifts to negative energies with respect to the Fermi level (i.e. $\Delta E=E_{D}-E_{F}<0 \mathrm{eV}$ ) which predicts $n$-doping for $\mathrm{Au}_{3} / \mathrm{G}$. In order to quantify $\Delta E$, we calculated and examined the band structure (see Supporting Note 3.1), which is more reliable than the PDOS for this purpose. This way, we retrieve $\Delta E=-54 \mathrm{meV}$ for $\mathrm{Au}_{3} / \mathrm{G}$, from which a doping efficiency of $\varepsilon_{\mathrm{Au}_{3}}^{\mathrm{th}} \approx 0.004 \mathrm{e} /$ cluster can be estimated. Note that both the sign and amplitude compare well to the experimentally retrieved doping efficiency in Fig. $2 \mathrm{~b}: \varepsilon_{\mathrm{Au}_{3}}^{\exp }=(0.007 \pm 0.002) e /$ cluster. The agreement with the computational results supports our interpretation that this state 
of the device captures the $\mathrm{Au}_{3} / \mathrm{G}$ interaction. For $\mathrm{Au}_{6}$, computation predicts a very mild, almost negligible $p$-doping of $\Delta E=2 \mathrm{meV}$ or $\varepsilon \approx 0.000006 \mathrm{~h} /$ cluster. This agrees with the closed electronic shell structure, and hence more inert chemical behaviour, of the twodimensional $\mathrm{Au}_{6} \cdot{ }^{[50]}$

While the experimentally observed doping efficiency will reflect an average charge transfer, with contributions from different isomers weighted by their population, we expect in this case that $\varepsilon_{\mathrm{Au}_{3,6}}^{\mathrm{th}}$ are close to the above discussed values based on the lowest-energy-isomer. Indeed, the four possibly coexisting $\mathrm{Au}_{6} / \mathrm{G}$ configurations (see Supporting Note 3.4) have an almost identical Fermi level shift $(\Delta E=1-2 \mathrm{meV})$, and the second-lowest-energy configuration for $\mathrm{Au}_{3} / \mathrm{G}(\Delta E=-10 \mathrm{meV})$ has and Au-cluster adhesion energy which is 50 meV higher than the lowest energy solution, so its population is expected to be low.

Figure 3e additionally shows the PDOS of $\mathrm{Au}_{3}$ and $\mathrm{Au}_{6}$ on graphene with $\mathrm{O}_{2}$ molecules. It is clear that both gold cluster - oxygen complexes result in significant $p$-doping: $\Delta E=145$ meV or $0.03 \mathrm{~h} /$ cluster for $\mathrm{Au}_{3}$. The computation for $\mathrm{Au}_{3}$ agrees well with the experiment presented in Fig. 2d: the sign of doping changes from $n$ to $p$ when oxygen is introduced. For $\mathrm{O}_{2} / \mathrm{Au}_{6} / \mathrm{G}$, the $p$-doping is found to be $\Delta E=370 \mathrm{meV}$ or $0.19 \mathrm{~h} /$ cluster. Quantitative comparison to experiments on $\mathrm{O}_{2} / \mathrm{Au}_{3,6} / \mathrm{G}$ devices are given below. Similar to the argument made for $\mathrm{Au}_{3,6} / \mathrm{G}$, we demonstrate in Supporting Note 3.6 that although more than one isomer of $\mathrm{O}_{2} / \mathrm{Au}_{3,6} / \mathrm{G}$ might coexist based on their relative $\mathrm{O}_{2}$ adsorption energies, the expected Fermi level shift is close to the value based on the lowest-energy solution for both $\mathrm{Au}_{3}$ and $\mathrm{Au}_{6}$.

The observed graphene doping and oxygen activation is in agreement with charge density difference maps, presented in Fig. 3f-i. From the side views, it can be distinguished that graphene loses electrons (although for the $\mathrm{O}_{2} / \mathrm{Au}_{3} / \mathrm{G}$ systems, locally graphene gains some electrons), which is consistent with the PDOS in Fig. 3e and the observed $p$-doping from transport experiments (Fig. 2d and Fig.4 below). Furthermore, from the top view in Fig. 3f and $g$, it is clear that the oxygen molecules gain electrons. This results in the elongation and hence activation of the oxygen molecules in Fig. $3 \mathrm{c}$ and d. The two $\mathrm{O}_{2}$ molecules closest to the $\mathrm{Au}_{3}$ cluster have a higher electron gain and hence a longer $\mathrm{O}-\mathrm{O}$ bond length than the molecules further away from the cluster (see Supporting Note 3.3 for quantification of the charge transfer through Bader analysis).

Due to the low flux achieved for $\mathrm{Au}_{6}$ clusters (see e.g. Fig. 1a), decelerating them during 
deposition leads to unmeasurable fluxes making it impossible to repeat a similar experiment as presented in Fig. 2. In order to investigate the effect of the deposited density $n_{c}$ and the cluster size $\left(\mathrm{Au}_{3}\right.$ and $\left.\mathrm{Au}_{6}\right)$ on the graphene doping, the following scheme was used: on different GFET devices, depositions were carried out without repelling the clusters, but rather attracting them in order for them to have a similar impact energy $\sim 22 \mathrm{eV}$. After each deposition, the devices were exposed to air. This removes any undesired effect induced by the impact of the charge particles and saturates the Au-cluster sites with oxygen. Using this procedure, subsequent depositions of $\mathrm{Au}_{6}^{-}$and $\mathrm{Au}_{3}^{+,-}$clusters were performed on eight separate devices. The result is depicted in Fig. 4, where the shift in charge neutrality point $\Delta V_{C N P}$ (doping) is shown with respect to the virgin state of the device, as function of the deposited cluster density. The agreement between the experiments performed with $\mathrm{Au}_{3}$ clusters on five seperate devices, illustrates that the difference in deposition energy and the charge of cluster ions in the employed beam does not alter the final result. The induced doping portrays a linear trend in function of $n_{c}$, as shown by the linear fits to the data, in agreement with charged impurity scattering theory in graphene. ${ }^{[51,52]}$ This linear trend demonstrates that, for the probed $n_{c}$ range, there is no transition to a regime where the adparticles undergo more coalescence. ${ }^{[21]}$ The currently presented systematic study of two distinct cluster sizes shows a non-trivial size effect, and evidences that single clusters are interacting with the graphene.

From Fig. 4, we estimate an average doping efficiency of $\varepsilon_{\mathrm{O}_{2} / \mathrm{Au}_{3}}^{\exp }=0.018 \pm 0.002 \mathrm{~h} / \mathrm{cluster}$ and $\varepsilon_{\mathrm{O}_{2} / \mathrm{Au}_{6}}^{\exp }=0.08 \pm 0.02 \mathrm{~h} /$ cluster for $\mathrm{Au}_{3}$ and $\mathrm{Au}_{6}$ clusters, respectively. Hence, the doping efficiency is clearly size-dependent in a non-trivial fashion. Indeed, the experimental doping efficiency of $\mathrm{O}_{2} / \mathrm{Au}_{6}$ complexes is a factor of $4.4 \pm 1.2$ larger than for $\mathrm{O}_{2} / \mathrm{Au}_{3}$. Even if one would define a doping efficiency as charge transfer per Au atom, there is a significant difference between the two cluster sizes. Our experimental findings are in good agreement with DFT computations of the $\mathrm{O}_{2} / \mathrm{Au}_{m} / \mathrm{G}$ systems in Fig. 3, where $p$-doping for both clusters sizes was retrieved and the doping efficiency of the system with $\mathrm{Au}_{6}$ is a factor of 6.3 larger than for $\mathrm{Au}_{3}$. As one would expect from charge impurity scattering theory, ${ }^{[51,52]}$ the the increase of charge carrier scattering also follows this distinct size-dependent charge transfer (see Supporting Note 4).

A side-by-side comparison of the experimental doping efficiencies and the ones obtained 


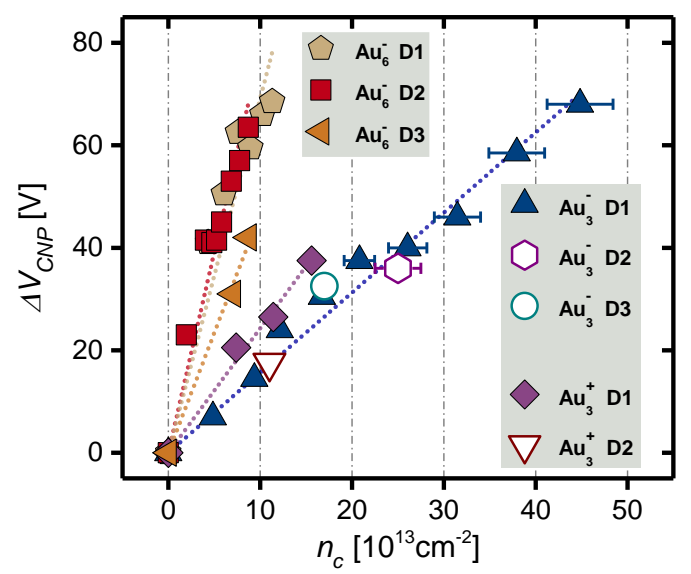

FIG. 4: Induced doping $\left(\Delta V_{C N P}\right)$ by $\mathrm{Au}_{3}$ - and $\mathrm{Au}_{6}$-oxygen complexes in function of the number of deposited clusters $n_{c}$. Data is shown for deposition runs on different devices. Error bars are omitted when smaller than symbol size. Linear fits to the data are represented with dotted lines. On the devices reported as $\mathrm{Au}_{3}^{-} \mathrm{D} 2$ and D3 and $\mathrm{Au}_{3}^{+}$D2, only one deposition was performed, and hence these points are without fit. All depositions were carried out at a deposition energy of $\sim 22 \mathrm{eV}$, except for $\mathrm{Au}_{3}^{-}$D3. The latter one is the experiment discussed in Fig. 2, performed at a deposition energy of $\mathrm{a} \sim 3 \mathrm{eV}$.

through DFT computation is presented in table I. The relative large error bar on the $\varepsilon_{A u_{6}}^{\exp }$ value can be attributed to the earlier discussed unwanted effects of the charge impact during cluster deposition. For $\mathrm{Au}_{3} / \mathrm{G}$ this uncertainty was reduced by softening the landing, which was less effective for $\mathrm{Au}_{6} / \mathrm{G}$ given its relatively lower flux.

TABLE I: Doping efficiency for $\left(\mathrm{O}_{2} /\right) \mathrm{Au}_{m} / \mathrm{G}$ with $m=3,6$.

\begin{tabular}{|c|c|c|}
\hline & $\varepsilon^{\exp }$ & $\varepsilon^{\text {th }}$ \\
\hline \hline $\mathrm{Au}_{3} / \mathrm{G}$ & $(0.007 \pm 0.002) e / \mathrm{cl}$ & $0.004 \mathrm{e} / \mathrm{cl}$ \\
\hline $\mathrm{Au}_{6} / \mathrm{G}$ & $(0.05 \pm 0.05) e / \mathrm{cl}$ & $0.000006 \mathrm{~h} / \mathrm{cl}$ \\
\hline $\mathrm{O}_{2} / \mathrm{Au}_{3} / \mathrm{G}$ & $(0.018 \pm 0.002) h / \mathrm{cl}$ & $0.03 \mathrm{~h} / \mathrm{cl}$ \\
\hline $\mathrm{O}_{2} / \mathrm{Au}_{6} / \mathrm{G}$ & $(0.08 \pm 0.02) h / \mathrm{cl}$ & $0.19 h / \mathrm{cl}$ \\
\hline
\end{tabular}

In conclusion, we realized $\mathrm{Au}_{3}$ and $\mathrm{Au}_{6}$ based graphene devices, and studied the sizedependent charge transfer and oxygen adsorption through FET measurements and DFT 
computations. We distinguished a non-trivial dependence on the cluster size. Suprisingly the oxygen adsorption energy on the few atom gold clusters is significanly enhanced by the presence of the graphene support.

As such, we demonstrated the usefulness of graphene as an in situ sensor for charge transfer. Indeed, when oxygen adsorbs to the Au-cluster sites, which itself results in $n$-doping, electrons transfer to the $\mathrm{O}_{2}$ molecules, leading to $p$-doping. This agrees with e.g. gas phase studies of oxygen adsorption on neutral Au clusters ${ }^{[40]}$, and this charge transfer, now in situ electronically probed through graphene, results in the activation of oxygen, which is a key mechanism in oxidation reactions in heterogeneous catalysis. Furthermore, this work demonstrates that cluster size-specific effect can be resolved in graphene, which provides perspectives to further explore clusters as ways to tailor desired properties in cluster-graphene hybrid systems.

\section{Acknowledgements}

The authors thank M. Houssa (KU Leuven) for fruitful discussions. This work is supported by the KU Leuven Internal Research Fund C14/17/080. H.-P.C. acknowledges the US DOE/BES DE-FG02-02ER45995 for supporting the computational work. J.-F.C. is Senior Research Associate of FRS-FNRS. Part of the research leading to these results received funding from the European Union H2020 program "Graphene Driven Revolutions in ICT and Beyond" (Graphene Flagship), project No 649953.

\section{Author contributions}

J.V.d.V. and E.J. initiated the research on size-selected clusters with graphene. J.E.S. and N.R. fabricated the graphene devices. J.E.S. performed the experiments and data analysis. V.S.Z. contributed to the cluster deposition and in situ gas experiments. S.L. and H.-P.C. were responsible for the computational part. J.E.S., J.V.d.V. and E.J. prepared the first version of the manuscript. All authors contributed to the data interpretation and participated in writing the manuscript.

\section{Experimental Section \\ Size-selected cluster production}

Size-selected $\mathrm{Au}_{3}$ and $\mathrm{Au}_{6}$ clusters are produced by magnetron sputtering and inert gas 
condensation. The sputter source is liquid nitrogen cooled, operates in continuous mode, and produces a large fraction of charged clusters. The (either cationic or anionic) clusters are mass-selected using a radiofrequency quadrupole mass filter with a maximum resolution of $m / \Delta m=200$. Deposition takes place in a UHV vacuum chamber (base pressure $10^{-10}$ mbar) in which the devices can be mounted for electronic measurements. The amount of deposited clusters is estimated by monitoring the beam current during deposition. A beam probe is used to determine the kinetic energy distribution of the deposited clusters.

\section{Device fabrication}

Single-layer graphene is grown on copper foil using a CVD procedure as described in Ref. 44. After growth, $5 \mathrm{~mm} \times 5 \mathrm{~mm}$ sheets are transferred onto a $7 \mathrm{~mm} \times 7 \mathrm{~mm} p^{++} \mathrm{Si} / \mathrm{SiO}_{2}$ substrate in a wet transfer procedure. Two double layer resist masks (PMMA/MA and PMMA 950K) are fabricated using a customized electron beam lithography platform from Raith GmbH: one to define the contact structures and one to define an etch mask. After writing the first mask, the titanium adhesion layer $(5 \mathrm{~nm})$ and gold $(30 \mathrm{~nm})$ are evaporated using a molecular beam epitaxy system at evaporation speeds of $1 \AA^{-1}$ and $0.23 \AA s^{-1}$, respectively. Reactive ion etching is performed after writing the second etch mask, in order to define the graphene transport channel. Each lithography step is followed by lift-off in acetone.

\section{GFET measurements and analysis}

FET measurements are performed in situ in the deposition chamber of the cluster apparatus, by applying a DC current of $I=1 \mu \mathrm{A}$ via the broad outer electrodes. The voltage drop $U$ over the sample is monitored using the voltage probes (see inset to Fig. 1b). From this, the sheet conductance $\sigma_{S}=\frac{I}{U} \frac{L}{W}$ in function of the applied gate voltage $V_{g}$ is retrieved. In order to retrieve the carrier-independent charge carrier mobility $\mu$, attributed to long-range Coulomb scatterers and a single characteristic scattering time, the Drude model is invoked. For the fit procedure, an interval of $\pm 1 \mathrm{~V}$ is taken around the steepest part of the slope $\left|d \sigma_{s} / d V_{g}\right|_{\text {max }}$, as is done in earlier works. ${ }^{[21,52,53]}$

\section{In situ gas exposure}

For in situ gas exposure, a gas inlet system with a regulating leak valve is present in the 
deposition chamber of the cluster apparatus. Samples are exposed at a constant effective pressure of $10^{-4}$ mbar. A Balzers Pfeiffer quadrupole mass spectrometer monitors the composition of the (rest) gas in the deposition chamber. During gas exposure with $\mathrm{O}_{2}$ (99.5\%) and $\operatorname{Ar}(99.9 \%)$, the $V_{C N P}$ was monitored (see Supporting Note 2).

\section{Computational details}

First-principles DFT calculations were performed utilizing the Vienna ab initio simulation package (VASP) ${ }^{[54,55]}$ with plane-wave cutoff energy set to $400 \mathrm{eV}$. Projector augmented wave potentials ${ }^{[56]}$ together with the generalized gradient approximation for the exchangecorrelation energy functional were used in all calculations. Van der Waals interactions are included via the optB86b functional ${ }^{[57-60]}$. The choice of this functional was motivated a thorough investigation of the dependence of the DFT functional on the obtained results for Au-clusters on graphene. ${ }^{[34]}$ In Ref. 34 it was found that long-range interactions are crucial for the binding of Au clusters to graphene and that the use of a non-local correlation functional, which (approximately) accounts for dispersion interactions is advised. The Fermi level shift $\Delta E$ and the adsorption of a single $\mathrm{O}_{2}$ molecule, calculated with different DFT functionals (see Supporting Note 3.6), provides insight in the dependence of the computational results on the selected functional. An energy criterion of $10^{-5} \mathrm{eV}$ was adopted for self-consistent field convergence. Structural relaxations were carried out until the Hellmann-Feynman force on each atom is less than $0.01 \mathrm{eV} / \AA$. Samplings of $5 \times 5 \times 1$ and $10 \times 10 \times 1 k$-points in the Brillouin zone were employed for ionic relaxation and charge analysis, respectively. An adaptive $k$-mesh, i.e. $125 \times 125 \times 1$ around the Dirac point and $25 \times 25 \times 1$ elsewhere in the reciprocal space, was employed to calculate the PDOS. The smearing parameter $\sigma$ was set to $0.01 \mathrm{eV}$ to capture the spin polarization of the gold-oxygen complexes. Zero point energy (ZPE) corrections are taken into account for all binding energies quoted in the main text.

\section{Calculation of doping efficiency $\varepsilon^{\text {exp }}$ from the experimental CNP shift}

From the induced CNP shift $\Delta V_{C N P}=V_{C N P}-V_{C N P o}$ in function of the deposited clusters, one can estimate the efficiency of the doping $\varepsilon^{\exp }$ in e/cluster (i.e. the amount of electrons donated to graphene) for $n$-doping, or in $\mathrm{h} /$ cluster for $p$-doping. We employ the relation between the shift in charge neutrality point and the induced density of charge carriers, 
$\Delta n=\alpha \Delta V_{C N P}$, where $\alpha=7.2 \cdot 10^{10} \mathrm{~V}^{-1} \mathrm{~cm}^{-2}$ as retrieved from the capacitance of the graphene $/ \mathrm{SiO}_{2} / p^{++}$stack. ${ }^{[46]}$ The doping efficiency is then defined as $\varepsilon^{\exp }=\Delta n / n_{c}^{\dagger}$, with $n_{c}^{\dagger}$ the estimated effective cluster density. For $\Delta V>0 \mathrm{~V}(\Delta V<0 \mathrm{~V})$, there is $p$-doping (n-doping). For devices on which subsequent clusters depositions were made, i.e. multiple $\Delta V_{C N P}\left(n_{c}\right)$ data points, one can estimate the experimental doping efficiency from the slope $b\left[\mathrm{~V} \cdot \mathrm{cm}^{2}\right]$ of the linear (least-squares) fits of $\Delta V_{C N P}$ as a function of $n_{c}: \varepsilon^{\exp }=\alpha \cdot b /\left(n_{c}^{\dagger} / n_{c}\right)$.

\section{Calculation of doping efficiency $\varepsilon^{\text {comp }}$ from computational band structure}

Computation of the band structures in Fig. 3 is carried out for a single cluster(-complex) within a $14.77 \AA \times 14.77 \AA$ unit cell, hence the simulated cluster density corresponds to $n_{c}^{\dagger}=5.29 \cdot 10^{13} \mathrm{~cm}^{-2}$. The induced density of charge carriers $\Delta n$, and hence the doping efficiency $\varepsilon^{\text {th }}=\Delta n / n_{c}^{\dagger}$, can be estimated from the Fermi shift with respect to the Dirac point $\Delta E=E_{\text {Dirac }}-E_{F}$. For pristine graphene, $\Delta E=0 \mathrm{eV}$, we employ graphene's linear dispersion relation $E(\vec{k})=\hbar v_{F} \vec{k}$, with $\vec{k}$ the 2 D-wavector and $v_{F} \sim 10^{8} \mathrm{~cm} / \mathrm{s}$ the Fermi velocity, ${ }^{[61]}$ to calculate the induced carrier density, provided that these dopants do not alter graphene's Dirac cone structure significantly. Since the Fermi level can be written as $E_{F}=E\left(\overrightarrow{k_{F}}\right)$ with $\left|\overrightarrow{k_{F}}\right|=\sqrt{\pi \Delta n}$ the Fermi wave vector, ${ }^{[61]}$ the doping efficiency is:

$$
\varepsilon^{\text {th }}=\frac{\Delta n}{n_{c}^{\dagger}}=\frac{\Delta E^{2}}{n_{c}^{\dagger} \pi \hbar^{2} v_{F}^{2}}=\Delta E^{2}\left[\mathrm{in} \mathrm{meV}^{2}\right] \cdot 1.39 \cdot 10^{-6} \frac{e, h}{\text { cluster }}
$$

\section{Supporting information}

The effect of the impact energy of the deposited charged clusters on graphene is described in Supporting Note 1. Gas exposure experiments with $\mathrm{Ar}$ and $\mathrm{O}_{2}$ to virgin devices are described in Supporting Note 2. More DFT results, namely the dispersion relations, the influence of the number of $\mathrm{O}_{2}$ molecules, a Bader analysis of the $\left(\mathrm{O}_{2}\right) / \mathrm{Au}_{3,6} / \mathrm{G}$ systems, a comparison of possible $\mathrm{Au}_{3,6}$ adsorption sites, detailed structural information, and the influence of the used DFT functional on the oxygen adsorption energy and Fermi level shift, are presented in Supporting Note 3. The graphene charge carrier mobility in function of $n_{c}$ 
for $\mathrm{Au}_{3,6}$, resulting in size-dependent induced scattering is discussed in Supporting Note 4.

* Electronic address: joris.vandevondel@kuleuven.be

$\dagger$ Electronic address: ewald.janssens@kuleuven.be

[1] K. S. Novoselov, A. K. Geim, S. V. Morozov, D. Jiang, Y. Zhang, S. V. Dubonos, I. V. Grigorieva, A. A. Firsov. Science 2004, 306666.

[2] C. R. Dean, A. F. Young, I. Meric, C. Lee, L. Wang, S. Sorgenfrei, K. Watanabe, T. Taniguchi, P. Kim, K. L. Shepard, J. Hone. Nat. Nanotechnol. 2010, 5722.

[3] W. Han, R. K. Kawakami, M. Gmitra, J. Fabian. Nat. Nanotechnol. 2014, 9794.

[4] D. Deng, K. S. Novoselov, Q. Fu, N. Zheng, Z. Tian, X. Bao. Nat. Nanotechnol. 2016, 11 218.

[5] A. K. Geim, I. V. Grigorieva. Nature 2013, 499419.

[6] T. Lee, S. H. Min, M. Gu, Y. K. Jung, W. Lee, J. U. Lee, D. G. Seong, B.-S. Kim. Chem. Mater. 2015, 273785.

[7] J. S. Bunch, A. M. van der Zande, S. S. Verbridge, I. W. Frank, D. M. Tanenbaum, J. M. Parpia, H. G. Craighead, P. L. McEuen. Science 2007, 315490.

[8] C. Chen, S. Rosenblatt, K. I. Bolotin, W. Kalb, P. Kim, I. Kymissis, H. L. Stormer, T. F. Heinz, J. Hone. Nat. Nanotechnol. 2009, 4861.

[9] P. Weber, J. Güttinger, A. Noury, J. Vergara-Cruz, A. Bachtold. Nat. Commun. 2016, 7 12496.

[10] F. Schedin, A. K. Geim, S. V. Morozov, E. W. Hill, P. Blake, M. I. Katsnelson, K. S. Novoselov. Nat. Mater. 2007, 6652 .

[11] S. S. Varghese, S. Lonkar, K. K. Singh, S. Swaminathan, A. Abdala. Sens. Actuator B-Chem. 2015, 218160.

[12] C. R. Woods, L. Britnell, A. Eckmann, R. S. Ma, J. C. Lu, H. M. Guo, X. Lin, G. L. Yu, Y. Cao, R. V. Gorbachev, A. V. Kretinin, J. Park, L. A. Ponomarenko, M. I. Katsnelson, Y. N. Gornostyrev, K. Watanabe, T. Taniguchi, C. Casiraghi, H.-J. Gao, A. K. Geim, K. S. Novoselov. Nat. Phys. 2014, 10451.

[13] L. Lin, L. Liao, J. Yin, H. Peng, Z. Liu. Nano Today 2015, 10701.

[14] S. Sarkar, M. L. Moser, X. Tian, X. Zhang, Y. F. Al-Hadeethi, R. Haddon. Chem. Mater. 
2014, 26184.

[15] E. C. Tyo, S. Vajda. Nat. Nanotechnol. 2015, 10577.

[16] R. Bhattacharjee, A. Datta. J. Phys. Chem. C 2017, 12120101.

[17] X. Gong, G. Liu, Y. Li, D. Y. W. Yu, W. Y. Teoh. Chem. Mater. 2016, 288082.

[18] B. Alfano, T. Polichetti, M. Mauriello, M. L. Miglietta, F. Ricciardella, E. Massera, G. Di Francia. Sens. Actuator B-Chem. 2016, 222 1032.

[19] D. Van Tuan, J. M. Marmoleja-Tejada, X. Waintal, B. K. Nikolić, S. O. Valenzuela, S. Roche. Phys. Rev. Lett. 2016, 117, 17176602.

[20] D. Sander, S. O. Valenzuela, D. Makarov, C. H. Marrows, E. E. Fullerton, P. Fisher, J. McCord, P. Vavassori, S. Mangin, P. Pirro, B. Hillebrands, A. D. Kent, T. Jungwirth, O. Gutfleisch, C. G. Kim, A. Berger. J. Phys. D: Appl. Phys. 2017, 50363001.

[21] J. E. Scheerder, T. Picot, N. Reckinger, T. Sneyder, V. S. Zharinov, J.-F. Colomer, E. Janssens, J. Van de Vondel. Nanoscale 2017, 910494.

[22] Z. Luo, A. W. Castleman Jr., S. N. Khanna. Chem. Rev. 2016, 11614456.

[23] Z. Li, H.-Y. T. Chen, K. Schouteden, T. Picot, K. Houben, T.-W. Liao, C. Van Haesendonck, G. Pacchioni, P. Lievens, E. Janssens. Nano Lett. 2016, 163063.

[24] Z. Li, H.-Y. T. Chen, K. Schouteden, T. Picot, A. Hillion, G. Pacchioni, C. Van Haesendonck, P. Lievens, E. Janssens. Nano Res. 2017, 101832.

[25] S. Bals, S. Van Aert, C. P. Romero, K. Lauwaet, M. J. Van Bael, B. Schoeters, B. Partoens, E. Yücelen, P. Lievens, G. Van Tendeloo. Nat. Commun. 2012, 3897.

[26] Q. Li, D. Yin, J. Li, F. L. Deepak. J. Phys. Chem. C 2018, 1121753.

[27] J. Li, D. Yin, C. Chen, Q. Li, L. Lin, R. Sun, S. Huang, Z. Wang. J. Appl. Phys. 2015, 117 085303.

[28] A. Mayoral, D. A. Blom, M. M. Mariscal, C. Guiterrez-Wing, J. Aspiazu, M. Jose-Yacaman. Chem. Commun. 2010, 468758.

[29] A. Sanchez, S. Abbet, U. Heiz, W.-D. Schneider, H. Häkkinen, R. N. Barnett, U. Landman. J. Phys. Chem. A. 1999, 1039573.

[30] B. Yoon, H. Häkkinen, U. Landman, A. S. Wörz, J.-M. Antonietti, S. Abbet, K. Judai, U. Heiz. Science 2005, 307403.

[31] A. A. Herzing, C. J. Kiely, A. F. Carley, P. Landon, G. J. Hutchings. Science 2008, 3211331.

[32] C. Liu, B. Yang, E. Tyo, S. Seifert, J. DeBartolo, B. von Issendorff, P. Zapol, S. Vajda, L. A. 
Curtiss. J. Am. Chem. Soc. 2015, 1378676.

[33] M. Shibuta, T. Ohta, M. Nakaya, H. Tsunoyama, T. Eguchi, A. Nakajima. J. Am. Chem. Soc. 2015, 13714015.

[34] M. K. Srivastava, Y. Wang, A. F. Kemper, H.-P. Cheng. Phys. Rev. B 2012, 85165444.

[35] G. Li, F. Li, X. Wang, M. Zhao, X. Liu. Phys. E 2014, 59235.

[36] W. Song, M. Jiao, K. Li, Y. Wang, Z. Wu. Chem. Phys. Lett. 2013, 588203.

[37] M. Haruta, T. Kobayashi, H. Sano, N. Yamada. Chem. Lett. 1987, 16405.

[38] B. Yoon, H. Häkkinen, U. Landman. J. Phys. Chem. A 2003, 1074066.

[39] A. P. Woodham, G. Meijers, A. Fielicke. Angew. Chem. Int. Ed. 2012, 514444.

[40] A. P. Woodham, G. Meijers, A. Fielicke. J. Am. Chem. Soc. 2013, 1351727.

[41] M. Gao, D. Horita, Y. Ono, A. Lyalin, S. Maeda, T. Taketsugu. J. Phys. Chem. C 2017, 121 2661.

[42] R. García-Cruz, E. Poulain, I. Hernández-Pérez, J. A. Reyes-Nava, J. C. González-Torres, A. Rubio-Ponce, O. Olvera-Neria. J. Phys. Chem. A 2017, 1216079.

[43] D. Schooss, P. Weis, O. Hampe, M. M. Kappes. Phil. Trans. R. Soc. A 2010, 3681211.

[44] N. Reckinger, X. Tang, F. Joucken, L. Lajaunie, R. Arenal, E. Dubois, B. Hackens, L. Henrard, J.-F. Colomer. Nanoscale 2016, 818751.

[45] K. S. Novoselov, A. K. Geim, S. V. Morozov, D. Jiang, M. I. Katsnelson, I. V. Grigorieva, S. V. Dubonos, A. A. Firsov. Nature 2005, 438197.

[46] K. Pi, K. M. McCreary, W. Bao, W. Han, Y. F. Chiang, Y. Li, S.-W. Tsai, C. N. Lau, R. K. Kawakami. Phys. Rev. B 2009, 80075406.

[47] G. M. Francis, I. M. Goldby, L. Kuipers, B. von Issendorff, R. E. Palmer. J. Chem. Soc., Dalton Trans. 1996, 0665.

[48] J. Wang, G. Wang, J. Zhao. Phys. Rev. B 2002, 66035418.

[49] Y.-N. Wu, M. Schmidt, J. Leygneir, H.-P. Cheng, A. Masson, C. Bréchignac. J. Chem. Phys. 2012, 136024314 .

[50] E. Janssens, H. Tanaka, S. Neukermans, R. E. Silverans, P. Lievens. New J. Phys. 2003, 5 46.

[51] S. Adam, E. H. Hwang, V. M. Galitski, S. Das Sarma. Proc. Natl. Acad. Sci. 2007, 104, 47 18392.

[52] J. H. Chen, C. Jang, S. Adam, M. S. Fuhrer, E. D. Williams, M. Ishigami. Nature Phys. 2008, 
4377.

[53] V. E. Dorgan, M.-H. Bae, E. Pop. Appl. Phys. Lett. 2010, 97082112.

[54] G. Kresse, J. Hafner. Phys. Rev. B 1993, 47558.

[55] G. Kresse, J. Furthmüller. Phys. Rev. B 1996, 5411169.

[56] P. E. Blöchl. Phys. Rev. B 1994, 5017953.

[57] J. Klimeš, D. R. Bowler, A. Michaelides. J. Phys. Cond. Matt. 2010, 22022201.

[58] J. Klimeš, D. R. Bowler, A. Michaelides. Phys. Rev. B 2011, 83195131.

[59] M. Dion, H. Rydberg, E. Schröder, D. C. Langreth, B. I. Lundqvist. Phys. Rev. Lett. 2004, 92 246401.

[60] G. Román-Pérez, J. M. Soler. Phys. Rev. Lett. 2009, 103096102.

[61] S. Das Sarma, S. Adam, E. H. Hwang, E. Rossi. Rev. Mod. Phys. 2011, 83407. 